\title{
Una mirada al IPSA: ¿Dónde estamos y hacia dónde vamos?
}

Este artículo presenta una descripción del Índice de Precios Selectivo de Acciones (IPSA), su composición y su relevancia como el principal índice de rentabilidad del mercado bursátil chileno. A continuación, analiza los principales factores que influenciaron su evolución en el periodo 20152017, con particular atención al rally iniciado en 2016 y la volatilidad observada durante el periodo de elecciones presidenciales. Para concluir, se presentan algunas perspectivas de su evolución para el año 2018.

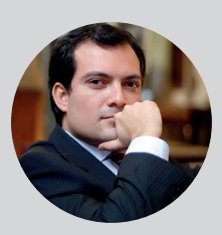

Andrés Román PORTFOLIO MANAGER, PROFESOR DE FINANZAS, UNIVERSIDAD ALBERTO HURTADO MSC. IN FINANCE, UNIVERSITY OF MANCHESTER, INGLATERRA INGENIERO COMERCIAL, UNIVERSIDAD DE CHILE

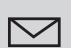

jroman@fen.uchile.cl

\section{¿Qué es y qué representa el IPSA?}

El Índice de Precios Selectivo de Acciones (IPSA) mide la rentabilidad promedio de las 40 acciones de mayor liquidez y tamaño en el mercado bursátil local. Este indicador es elaborado por la Bolsa de Comercio de Santiago y es el principal índice del mercado bursátil local. Otros índices considerados a nivel local son el Índice General de Precios de Acciones (IGPA) y MSCl Chile. El primero incluye, además de las empresas consideradas en el IPSA, a un grupo de empresas pequeñas que tienen un bajo nivel de liquidez. Este índice tiene una mayor cobertura pero sus precios son menos representativos del valor de las empresas que lo componen. Por su parte, el $\mathrm{MSCl}$ Chile se construye en base a una metodología que lo hace comparable con otros índices internacionales, pero considera un número reducido de empresas (19) y, por tanto, se encuentra menos diversificado y es menos representativo de la bolsa local que el IPSA.
La Tabla 1 muestra las empresas consideradas en el IPSA y su ponderación en el índice para el año 2017. Se aprecia que las empresas con mayor ponderación en el índice son Copec (8,8\%), Falabella $(6,8 \%), \operatorname{LTM}(6,8 \%)$ y ENELAM (6,7\%). En conjunto, las diez empresas de mayor ponderación explican un 58\% del índice. La Tabla 2 muestra su composición sectorial. Se aprecia que los sectores Commodities, Bancos, Utilities, y Retail representan $76,4 \%$ del índice. 
TABLA 1

\begin{tabular}{lclc}
\hline EMPRESAS & PONDERACIÓN & EMPRESAS & PONDERACIÓN \\
\hline COPEC & $8,8 \%$ & ENTEL & $1,7 \%$ \\
FALABELLA & $6,8 \%$ & CAP & $1,4 \%$ \\
LTM & $6,8 \%$ & ANDINA-B & $1,3 \%$ \\
ENELAM & $6,7 \%$ & BANMEDICA & $1,3 \%$ \\
CHILE & $5,7 \%$ & ECL & $1,2 \%$ \\
SQM-B & $5,5 \%$ & QUINENCO & $1,2 \%$ \\
BSANTANDER & $5,5 \%$ & RIPLEY & $1,2 \%$ \\
CENCOSUD & $4,4 \%$ & SONDA & $1,1 \%$ \\
CMPC & $4,2 \%$ & AESGENER & $1,0 \%$ \\
BCI & $3,8 \%$ & CONCHATORO & $0,9 \%$ \\
ENELGXCH & $3,3 \%$ & VAPORES & $0,9 \%$ \\
SM-CHILE B & $2,6 \%$ & IAM & $0,9 \%$ \\
ITAUCORP & $2,5 \%$ & ILC & $0,7 \%$ \\
ENELCHILE & $2,5 \%$ & SK & $0,6 \%$ \\
CCU & $2,4 \%$ & SMSAAM & $0,5 \%$ \\
ANTARCHILE & $2,3 \%$ & SALFACORP & $0,5 \%$ \\
PARAUCO & $2,3 \%$ & FORUS & $0,5 \%$ \\
COLBUN & $2,2 \%$ & EMBONOR-B & $0,4 \%$ \\
AGUAS-A & $2,1 \%$ & ORO BLANCO & $0,4 \%$ \\
SECURITY & $1,7 \%$ & MASISA & $0,2 \%$ \\
\hline
\end{tabular}

Fuente: Bolsa de Comercio de Santiago, diciembre del 2017

TABLA 2

\begin{tabular}{lc}
\hline SECTOR & PONDERACIÓN \\
\hline Commodities & $22,7 \%$ \\
Bancos & $21,7 \%$ \\
Utilities & $19,9 \%$ \\
Retail & $15,1 \%$ \\
Transporte & $8,2 \%$ \\
Bebidas & $5,1 \%$ \\
TI \& Telecomunicaciones & $2,7 \%$ \\
Construcción e inmobiliario & $1,4 \%$ \\
Otros & $3,2 \%$
\end{tabular}

Fuente: Bolsa de Comercio de Santiago, diciembre del 2017

\section{Composición sectorial del IPSA} pertenecen al IPSA y su ponderación

Empresas que

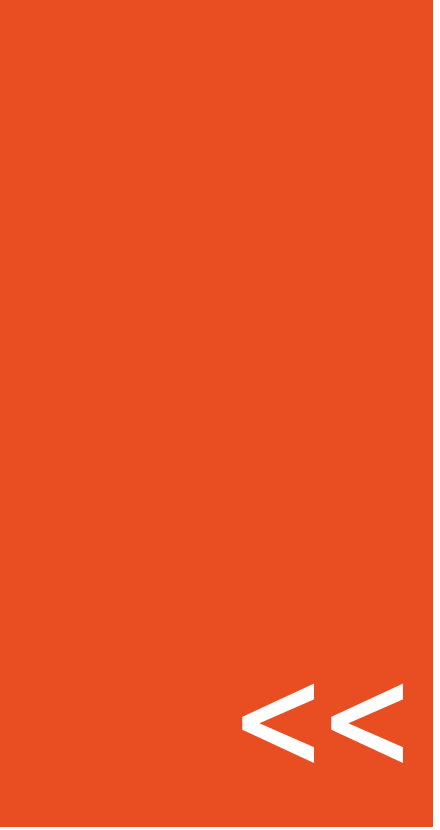




\section{¿Cuál es la relevancia del IPSA?}

En un mercado sin restricciones para la transacción de acciones, el precio de éstas refleja la disposición a pagar de los inversionistas por los flujos de caja que las empresas producirán en el futuro para sus dueños. En este contexto, el IPSA puede interpretarse como un termómetro respecto a las expectativas de generación de flujos de caja futuros por parte de las principales sociedades anónimas abiertas chilenas. Como las perspectivas sobre el desempeño empresarial están estrechamente relacionadas con las expectativas sobre nuestra economía, el IPSA también puede interpretarse como un termómetro que mide las expectativas sobre el desempeño de la economía local. En otras palabras, mientras mayor sea el dinamismo esperado de nuestra economía, mejores serán las expectativas sobre los resultados de las empresas y esto impactará positivamente en sus valorizaciones. En efecto, la mayor disposición a pagar de los inversionistas aumentará el precio de las acciones de manera individual y el promedio que mide el IPSA. Por el contrario, si las expectativas a futuro sobre la economía son débiles, es probable que este índice caiga debido a que los inversionistas tendrían una menor disposición a pagar por éstas.

\section{Evolución del IPSA entre 2015 y 2017}

Como se aprecia en el Gráfico 1, tras alcanzar un mínimo de 3.455 puntos el 20 de enero del año 2016, el IPSA comenzó una tendencia alcista alcanzando un máximo de 5.608 puntos el 30 de octubre del año 2017, representando un alza del $62,3 \%$ en dicho periodo. Posteriormente, el IPSA presentó una corrección de 12,8\% alcanzando los 4.887 puntos el día 7 de diciembre, para luego alcanzar los 5.565 puntos al cierre de 2017.

\section{GRÁFICO 1}

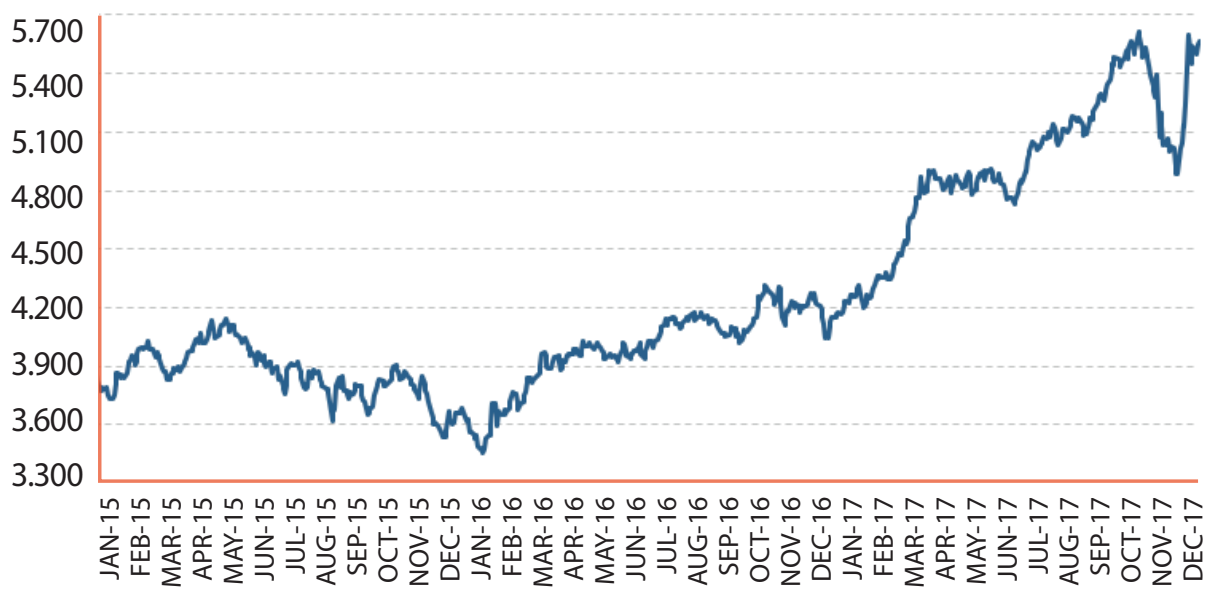

Fuente: Bolsa de Comercio de Santiago, diciembre de 2017

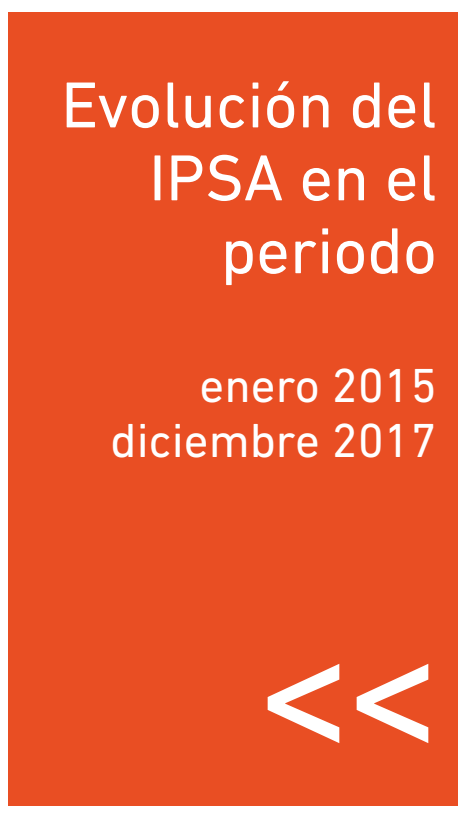

El principal catalizador del rally experimentado por el IPSA desde comienzos del año 2016 fue la mayor disposición a pagar de los inversionistas producto del alza en las expectativas de generación de flujos de caja futuros por parte de las principales sociedades anónimas abiertas chilenas. Dicha mejora sería consecuencia de un mayor dinamismo en la economía chilena que se explicaba por dos factores. Primero, un escenario externo más favorable influenciado por las bajas tasas de interés a nivel global, mayores expectativas de crecimiento de diversos países y un alza del precio de los commodities, en particular el cobre con estimaciones superiores a US\$3.0 la libra. Segundo, una dinámica interna más robusta debido a las expectativas de que un eventual gobierno de derecha sería más "pro crecimiento", impulsando la inversión en diversos sectores de la economía local. Esta mejora en las expectativas de crecimiento de la econo- mía chilena se vio reflejada en distintos indicadores de confianza. Por ejemplo, el Índice de Percepción Económica de los Consumidores (IPEC, Adimark) alcanzó 46,5 puntos en el mes de octubre, superando al mínimo de 31,5 puntos alcanzado en el mes de agosto de 2016. Del mismo modo, el Índice Mensual de Confianza Empresarial (IMCE, ICARE-UAI) alcanzó 49 puntos en octubre, comparándose positivamente con los 42,4 puntos alcanzados en el mes de julio del mismo año. 
TABLA 3

\begin{tabular}{lrr}
\hline ÍNDICES & CIERRE & $\mathbf{2 0 1 7}$ \\
\hline Chile (IPSA) & 5.565 & $34,0 \%$ \\
Brasil (Bovespa) & 76.402 & $26,9 \%$ \\
México (Mexbol) & 49.354 & $8,1 \%$ \\
Perú (SP/BVL Select) & 368,0 & $23,5 \%$ \\
Colombia (COLCAP) & 1.514 & $12,0 \%$ \\
Argentina (Merval) & 30.066 & $77,7 \%$ \\
\hline
\end{tabular}

Fuente: Bloomberg.

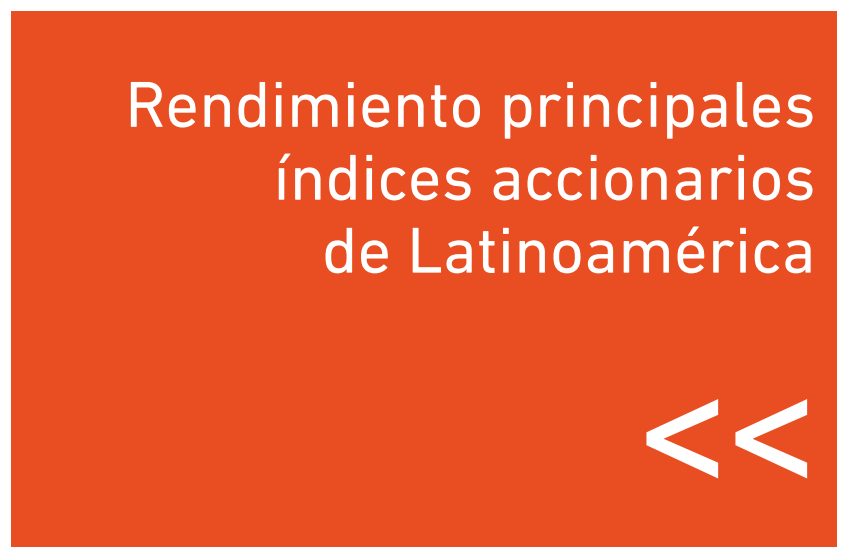

Similar rally han registrado los principales índices bursátiles de los países latinoamericanos durante el año 2017. tal como se aprecia en la Tabla 3. Las mejores expectativas de crecimiento de sus economías y sus resultados corporativos han sido los drivers que explican este buen rendimiento.

\section{IPSA y las elecciones de diciembre de 2017}

A fines del 2017, la bolsa local vivió uno de los periodos de mayor volatilidad de los últimos años. A juicio de varios analistas, esta volatilidad se debió a la incertidumbre respecto al resultado de las elecciones presidenciales. En efecto, durante el periodo precedente a la primera vuelta presidencial (19 de noviembre de 2017), el favoritismo de Piñera en las encuestas que lo daban como próximo presidente de Chile era uno de los principales motivos que impulsaban a los inversionistas a apostar por la bolsa local ante el llamado "cambio de expectativas" que se generaría, dado que el entonces candidato Piñera había dado mayores señales pro-mercado que el candidato Guillier ${ }^{1}$.

Sin embargo, el sorpresivo resultado de primera vuelta, que anticipaba una dura lucha entre Piñera y Guillier en el balotaje, trajo consigo un aumento en la incertidumbre y cautela en los inversionistas ${ }^{2}$. Esta incertidumbre se reflejó en una alta volatilidad en el mercado haciendo que muchos inversionistas, que no estaban dispuestos a soportar este mayor riesgo, salieran a vender sus acciones locales presionando el nivel del IPSA a la baja. Es así como vimos que el IPSA pasó de niveles de 5.608 puntos el 30 de octubre al mínimo 4.887 puntos el 8 de diciembre, representando una caída de 12,8\% en dicho periodo. Una vez conocido el resultado de la segunda vuelta, el mercado lo interpretó positivamente y el IPSA recuperó rápidamente los niveles máximos alcanzados a fines de mes de octubre.

\section{¿Cómo viene el IPSA en 2018?}

Para abordar esta pregunta, es conveniente considerar la razón $P / U$, que corresponde al ratio entre el precio de accionario (P) y la utilidad por acción de los últimos doce meses (U). Este ratio indica el precio que pagan los inversionistas por cada peso de utilidad que genera una empresa y se interpreta como una medida de que tan cara o barata se encuentra la bolsa local. A mayor ratio $\mathrm{P} / \mathrm{U}$, más cara se encuentra la bolsa local porque los inversores están pagando un mayor precio por cada peso de ganancia que producen las empresas.

Al considerar la valoración implícita del IPSA medido por el ratio P/U, este pareciera estar caro debido a que se transa con un múltiplo de 24,3 veces, cifra que excede a su promedio de los últimos 4 años (20 veces). Sin embargo, al considerar que las expectativas de crecimiento de las utilidades corporativas se sitúan en 16,3\% para el 2018 , unido al valor objetivo del IPSA esperado por el consenso de los analistas del mercado para fines del 2018³, el IPSA "esperado" se transaría a un múltiplo más ajustado de 22,3 veces, nivel algo superior a su media. Del mismo modo, las revisiones alcistas en utilidades que vendrán a partir del 2019 reducen aún más el valor esperado de este múltiplo. Las futuras revisiones alcistas de las utilidades de las empresas podrían ser suficientes para que el IPSA continúe con su trayectoria positiva. En otras palabras, existen altas probabilidades de que el dinamismo de la economía sea mejor a lo esperado, impactando también en el crecimiento de las utilidades de las empresas.

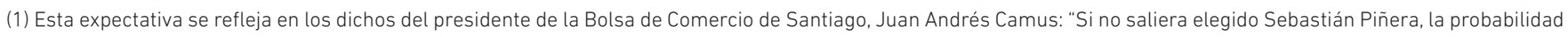
de que tengamos un colapso en el precio de las acciones es alta", que representaba la visión de diversos analistas.

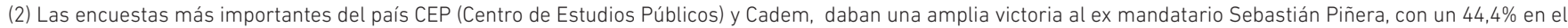

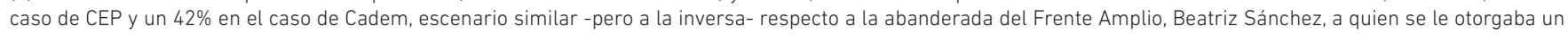

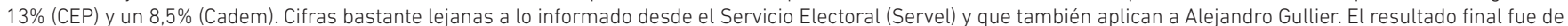
un $36,64 \%, 22,7 \%$ y $20,3 \%$ de los votos para Piñera, Guillier y Sánchez, respectivamente.

(3) El consenso de Bloomberg asigna un valor objetivo de 6.000 puntos a fines del 2018. 


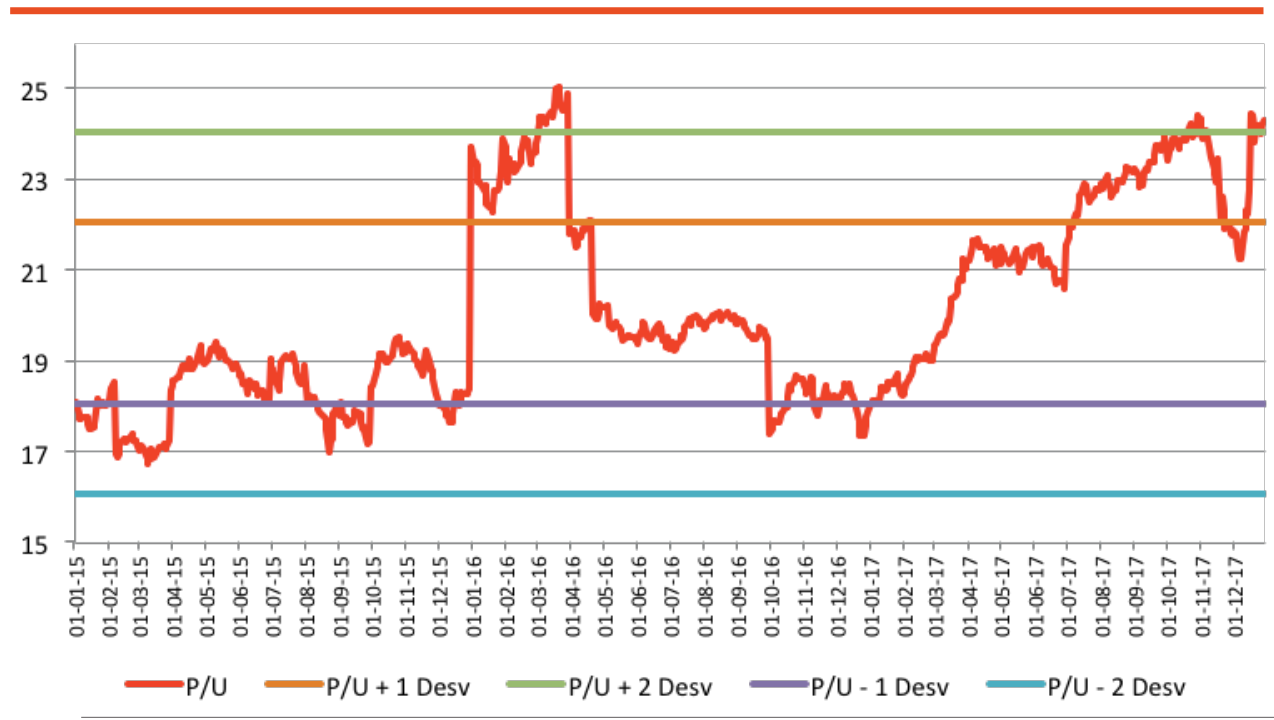

Fuente: Elaboración Propia con datos de Bloomberg

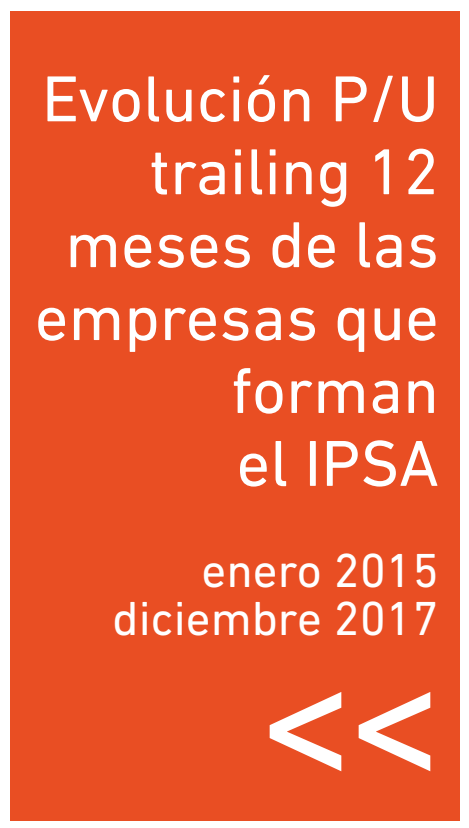

$C_{\text {Los }}$

indicadores

sugieren que el IPSA seguirá con la tendencia alcista iniciada el año 2016 9)

A nivel sectorial, las empresas de sectores más cíclicos como la banca, el retail y la construcción, que en conjunto ponderan un 45,8\% del IPSA, se verían particularmente beneficiadas por el mayor crecimiento del país. Específicamente, se estima que el sector bancario estará influenciado por un crecimiento en las colocaciones superior al registrado el año 2017, compensando la pérdida que pudieran tener por el hecho de que la inflación proyectada es inferior a la meta del 3,0\% del Banco Central. Del mismo modo, el aumento esperado en las ventas del sector retail, debido a la mayor propensión a gastar de las personas, sería un buen catalizador para que las tiendas por departamento registren utilidades mayores a las observadas en el 2017. El sector construcción también se vería beneficiado producto del aumento en la inversión pública y privada, que impulsarían los resultados de las empresas que componen este sector.

A nivel internacional, una desaceleración en China menor a la esperada y mayor crecimiento global deberían impulsar los precios de los commodities. Es así como la celulosa se mantendría en niveles de precios altos debido a una mayor demanda de este insumo y una oferta acotada en esta industria. El yodo y potasio, por su parte, se mantendrían con un sesgo alcista producto de su mayor demanda. El cambio estructural en la demanda por litio, debido a la irrupción de los vehículos eléctricos, unido a la creciente industria de baterías que usan este componente, indican que el precio de este commodity pudiera seguir alcanzando nuevos máximos.

El sector de servicios básicos, en especial el eléctrico, si bien pudiera mostrar crecimientos en utilidades más neutrales el año 2018, debido al cambio en la composición de su matriz energética incorporando un porcentaje cada vez mayor de energías renovables no convencionales, se encuentra con valoraciones atractivas al compararlas con su historia.

Todo lo anterior, indica que el IPSA seguirá con la tendencia alcista iniciada el año 2016, anticipando un escenario de utilidades corporativas positivo tanto para el año 2018 como para los años posteriores.

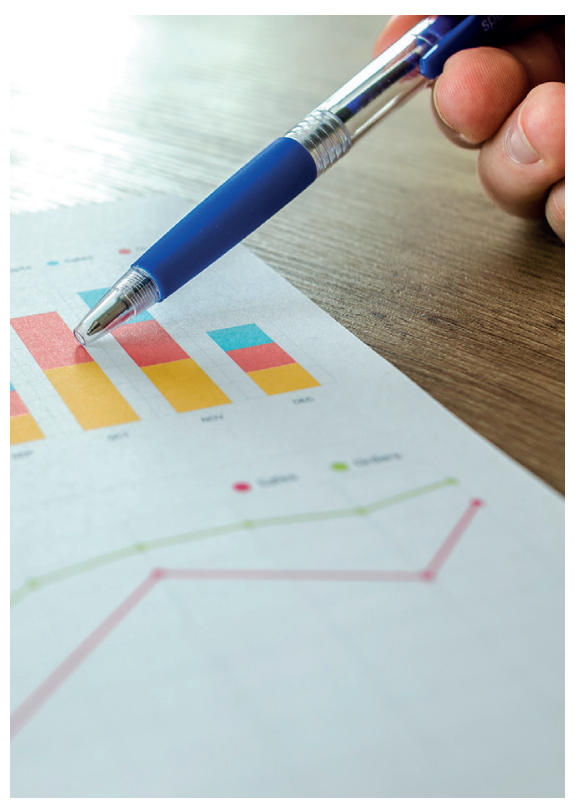

